\title{
Self-reported physical activity level does not alter whole- body total heat loss independently of aerobic fitness in young adults during exercise in the heat
}

\begin{tabular}{|c|c|}
\hline Journal: & Applied Physiology, Nutrition, and Metabolism \\
\hline Manuscript ID & apnm-2018-0211.R1 \\
\hline Manuscript Type: & Brief communication \\
\hline $\begin{array}{r}\text { Date Submitted by the } \\
\text { Author: }\end{array}$ & 06-Jun-2018 \\
\hline Complete List of Authors: & $\begin{array}{l}\text { Lamarche, Dallon; University of Ottawa, Human Kinetics } \\
\text { Notley, Sean R.; University of Ottawa, Faculty of Health } \\
\text { Sciences } \\
\text { Poirier, Martin; University of Ottawa, School of Human Kinetics, } \\
\text { Faculty of Health Sciences } \\
\text { Kenny, Glen P.; University of Ottawa, Human Kinetics }\end{array}$ \\
\hline $\begin{array}{r}\text { Is the invited manuscript } \\
\text { for consideration in a } \\
\text { Special Issue? : }\end{array}$ & Not applicable (regular submission) \\
\hline Keyword: & $\begin{array}{l}\text { heat loss, calorimetry, self-reported physical activity, peak } \\
\text { oxygen consumption, exercise < exercise }\end{array}$ \\
\hline
\end{tabular}


Self-reported physical activity level does not alter whole-body total heat loss independently of aerobic fitness in young adults during exercise in the heat

Dallon T. Lamarche, Sean R. Notley, Martin P. Poirier, and Glen P. Kenny

Human and Environmental Physiological Research Unit, Faculty of Health Sciences, University of Ottawa, Ottawa, Ontario, Canada, K1N 6N5

Running title: Self-reported physical activity \& heat dissipation

Exposition word count: 2311 (text, references)/3000

Number of references: 10

Address for correspondence:

Glen P. Kenny

125 University Private

Room 367, Montpetit Hall

Ottawa, Ontario, Canada, K1N 6N5

E-mail: gkenny@uottawa.ca

Phone: +1 613-562-5800 ext. 4282

Fax: +1 613-562-549 
Self-reported physical activity \& heat dissipation

\section{ABSTRACT (Word count: 75/75)}

We evaluated whether self-reported physical activity (PA) level modulates whole-body total heat loss (WB-THL) as assessed using direct calorimetry in ten young adults ( $22 \pm 3$ years) matched for rate of peak oxygen consumption $\left(\dot{\mathrm{V}}_{2 \text { peak }}\right.$; an index for aerobic fitness), but of low and high self-reported PA, during three incremental cycling bouts $\left(\sim 39,52\right.$, and $\left.64 \% \dot{\mathrm{V}}_{2 \text { peak }}\right)$ in the heat $\left(40^{\circ} \mathrm{C}\right)$. We showed that level of self-reported PA does not appear to influence WB-THL independently of $\dot{\mathrm{V}} \mathrm{O}_{2 \text { peak }}$.

Key words: self-reported physical activity level, peak oxygen consumption, exercise, heat loss, calorimetry 
Self-reported physical activity \& heat dissipation

\section{INTRODUCTION}

It has long been viewed that individuals with a high level of aerobic fitness (typically represented by higher training-induced rates of peak oxygen consumption $\left(\dot{\mathbf{V}} \mathbf{O}_{2 \text { peak }}\right)$ relative to body mass), tend to demonstrate a higher capacity to dissipate heat (Saltin and Hermansen 1966). This is evidenced by a greater whole-body sweat production in highly fit men and women compared to lesser trained counterparts during exercise in the heat (Lamarche et al. 2017a, 2017b). This difference is primarily thought to be mediated by frequent participation in intense and/or prolonged endurance exercise training that induces elevations in body temperature (Périard et al. 2015) and subsequent thermal and cardiovascular adaptations (e.g., increases in the number of active sweat glands and their output, increase in plasma volume, others). These adaptations are comparable to those observed during heat acclimation (Amano et al. 2013; Avellini et al. 1982; Henane et al. 1977) and are thought to partially explain the improved sweating response observed in higher aerobic fitness individuals. Moreover, while $\dot{\mathrm{V}} \mathrm{O}_{2 \text { peak }}$ is often linked to an individuals' level of aerobic fitness, $\dot{\mathrm{V}} \mathrm{O}_{2 \text { peak }}$ is, in part, genetically determined (Bouchard et al. 1999). As such, individuals with large differences in their PA level can possess a similar $\dot{\mathrm{VO}}_{2 \text { peak. }}$ It is therefore possible that individuals who regularly perform intense and/or prolonged physical activity (PA) may possess training-induced adaptations that increase wholebody sweating, and thus whole-body total heat loss (WB-THL), relative to those who perform

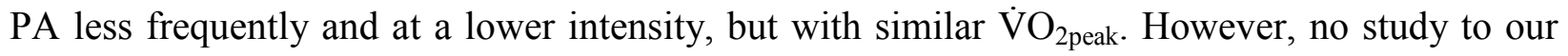
knowledge has been designed to evaluate WB-THL during exercise in the heat between individuals possessing large differences in their level of self-reported PA (as defined by intensity

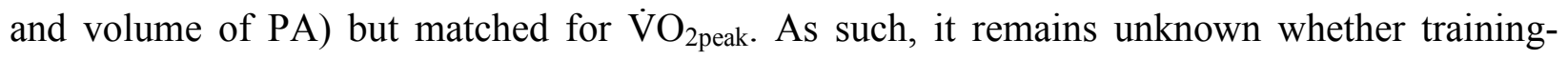


Self-reported physical activity \& heat dissipation

related improvements in WB-THL can occur independently of an increase in $\dot{\mathrm{V}} \mathrm{O}_{2 \text { peak }}$ as both physiological outcomes can be mediated by the level of exercise training.

We therefore examined changes in WB-THL, as assessed by direct calorimetry, in healthy young adults matched for $\dot{\mathrm{V}} \mathrm{O}_{2 \text { peak }}$, but with low and high levels of self-reported PA (assessed using questionnaires), during exercise in the heat eliciting equivalent combined metabolic and environmental (net) heat loads and therefore requirements for WB-THL. Since our previous work has shown that aerobic fitness modulates WB-THL as a function of increasing exercise intensity (Lamarche et al. 2017a, 2017b), these groups were assessed during exercise performed at $\sim 39,52$ and $64 \%$ of $\dot{\mathrm{V}}_{2 \text { peak. In addition, secondary factors known to modulate heat }}$ loss (e.g., body mass, surface area, adiposity, age) were carefully matched between groups. We hypothesized that WB-THL would be greater in individuals with higher self-reported PA as compared to their lesser active counterparts, with the magnitude of those differences being greater at higher net heat loads.

\section{METHODS}

\section{Ethical approval}

The experimental protocol was approved by the University of Ottawa Health Sciences and Science Research Ethic Board and agrees with the Declaration of Helsinki. Written informed consent was obtained from all volunteers prior to their participation in the study.

\section{Participants}

Twenty healthy, non-smoking young (18-to-30 years) men $(n=6)$ and women $(n=14)$ participated (Table I). Participants were selected based on their $\dot{\mathrm{VO}}_{2 \text { peak, }}$, self-reported PA levels, and physical characteristics and separated into two groups of low and high PA: Low-PA and 
Self-reported physical activity \& heat dissipation

High-PA (each $n=10$, with three men and seven women within each group). Self-reported PA was assessed by two validated questionnaires (Kohl et al. 1988; Baecke et al. 1982). The Baecke Sport Index questionnaire was used to calculate a sport index score (5-point scale, with 5 being the most physically active) as well as the intensity (low, medium, high), and volume (minutes per week) of PA performed. Individuals scoring $\leq 3$ were considered Low-PA and $\geq 4$ as High-PA. The Kohl PA questionnaire was used to confirm the volume of PA noted in the Baecke questionnaire and to provide more detail about the type of PA performed. This approach ensured that the High-PA performed $\geq 300$ minutes per week of running, cycling, and/or cross-country skiing at a moderate-to-high intensity. These participants were each matched for physical characteristics and $\dot{\mathrm{V}} \mathrm{O}_{2 \text { peak }}$ with a Low-PA individual (performing $<200$ minutes per week of aerobic $\mathrm{PA})$.

\section{Experimental Design}

Participants completed one preliminary and one experimental session each separated by $\geq 48$ h. For all sessions, participants were instructed to arrive at the laboratory adequately rested and hydrated as well as abstained from exercise, caffeine and alcohol $24 \mathrm{~h}$ prior. During the preliminary session, body mass, height, density and $\dot{\mathrm{V}} \mathrm{O}_{2 \text { peak }}$ were determined. $\dot{\mathrm{V}} \mathrm{O}_{2 \text { peak }}$ was assessed during an incremental semi-recumbent cycling exercise test to volitional fatigue. Upon arrival to their experimental session, participants changed into athletic shorts (and sports bra for females) and provided a urine sample to confirm participants were similarly euhydrated $(<1.020)$ before having their body mass measured. Following instrumentation under thermoneutral conditions $\left(\sim 25^{\circ} \mathrm{C}\right)$, participants were seated in the calorimeter regulated to an ambient air temperature of $40^{\circ} \mathrm{C}$ and a relative humidity of $10 \%$ (specific humidity of $\sim 5 \mathrm{~g}$ water $\cdot \mathrm{kg}$ ambient 
Self-reported physical activity \& heat dissipation

air $^{-1}$ ) and rested for a 30-min habituation period. Thereafter, they performed three 30-min semirecumbent cycling bouts at $\sim 39 \%$ of $\dot{\mathrm{V}} \mathrm{O}_{2 \text { peak }}$ for $\mathrm{Ex} 1 ; 52 \%$ of $\dot{\mathrm{V}} \mathrm{O}_{2 \text { peak }}$ for Ex 2 and $64 \%$ of $\dot{\mathrm{V}} \mathrm{O}_{2 \text { peak }}$ for Ex3; each separated by a 15-min resting recovery (R1, R2, R3).

\section{Measurements}

The modified Snellen direct air calorimeter was used to perform continuous measurements of whole-body evaporative heat loss (WB-EHL) and dry heat exchange (WBDHE; dry heat gain in the conditions tested), and metabolic heat liberation was calculated by indirect calorimetry. Esophageal temperature was measured continuously using a thermocouple temperature probe (Mon-a-therm General Purpose Temperature Probe; Mallinckrodt Medical, St. Louis, MO, USA). Mean skin temperatures were assessed continuously using the weighted average of four sites: biceps $30 \%$, chest $30 \%$, thigh $20 \%$, and calf $20 \%$. Heart rate was recorded continuously (Polar Electro, Oy, Finland). Urine specific gravity was determined prior to each experimental trial using a hand-held refractometer (model TS400; Reichter, Depew, NY, USA).

\section{Data Analysis}

The change in body heat storage was calculated as the temporal summation of heat gain and WB-THL (WB-EHL \pm WB-DHE). WB-THL, WB-EHL and WB-DHE, esophageal and mean skin temperatures and heart rate were expressed as minute averages, with an average of the final 5-mins of each exercise period used for statistical analyses. Baseline values were obtained by averaging the last 10 min of data recorded during the 30 -min baseline period.

\section{Statistical Analysis}


Self-reported physical activity \& heat dissipation

Physical characteristics and baseline resting values were analyzed using an independent samples $t$-test to identify differences between groups. WB-THL, WB-EHL and WB-DHE, esophageal and mean skin temperatures, and heart rate were analyzed using a mixed two-way ANOVA with one factor of group [Low-PA and High-PA] and the repeated factor of exercise $(\mathrm{Ex} 1, \mathrm{Ex} 2, \mathrm{Ex} 3)$. Based on the effect size (Cohen's $d=1.88)$ for a mean difference of $32 \mathrm{~W}$ with a standard deviation of $\sim 17 \mathrm{~W}$ in WB-THL between high- and low-fit groups observed in our previous work (Lamarche et al. 2017a), 8 subjects per group would be required to detect differences between Low-PA and High-PA individuals of this effect size with $>80 \%$ statistical power. Therefore, with the current sample ( $n=10$ per group), these analyses were adequately powered. Statistical significance was set at $P<0.05$, while a Bonferroni adjustment was used for all post hoc comparisons. Data are reported as means $\pm 95 \%$ confidence intervals unless stated otherwise as SD. All analyses were completed using SPSS version 24.0 for Windows (IBM, Armonk, NY, USA).

\section{RESULTS}

Metabolic heat production, WB-EHL and WB-DHE were similar between the Low-PA and High-PA groups during the baseline and throughout exercise (Table II, all $P>0.05$ ). Consequently, WB-THL at baseline and during exercise, as well as the resulting change in body heat storage across all three exercise periods (excluding recovery) was similar between the LowPA and High-PA groups (Fig. I, all $P>0.05$ ). Esophageal and mean skin temperature as well as heart rate were also similar between groups at baseline and during exercise (Table II, all $P>0.05$ ).

\section{DISCUSSION}


Self-reported physical activity \& heat dissipation

We evaluated whether WB-THL, as assessed by direct calorimetry, was enhanced in young adults with high levels of self-reported PA relative to individuals with low levels of selfreported PA, but with matched $\dot{\mathrm{V}} \mathrm{O}_{2 \text { peak }}$ and physical characteristics. With this unique design, we could determine the influence of PA level on the body's physiological capacity to dissipate heat independently from the effects of differences in $\dot{\mathrm{V}} \mathrm{O}_{2 \text { peak }}$ on thermoregulatory function. In contrast to our working hypothesis, WB-THL and the subsequent changes in body heat storage during exercise were similar between groups, indicating that self-reported PA level does not appear to influence thermoregulatory function independently from $\dot{\mathrm{V}} \mathrm{O}_{2 \text { peak. This is important, as }}$ it remained uncertain as to whether physically inactive individuals with a similar $\dot{\mathrm{V}}_{2 \text { peak }}$ to more active individuals display impairments in whole-body heat dissipation during exercise or work in hot conditions.

Fitness-related improvements in whole-body sweat production have been ascribed, in part, to the fact that aerobically fit individuals generally perform regular endurance exercise training that induces elevations in body temperature and subsequent adaptations that enhance the sweating response (Amano et al. 2013; Avellini et al. 1982; Henane et al. 1977). Consequently, we surmised that individuals with a high level of self-reported PA would display enhanced WBTHL relative to individuals with low levels of self-reported PA, but similar $\mathrm{VO}_{2 \text { peak }}$. However, despite large differences in the intensity and frequency of self-reported PA (Table I), no differences in WB-THL and therefore changes in body heat storage (Fig. I) or body core temperature (Table II) were observed between the high and low PA groups. Combined with our previous study findings, where we observed improvements in heat loss among individuals with both a high $\dot{\mathrm{V}}{ }_{2 \text { peak }}$ and PA level (Lamarche et al. 2017a, 2017b), our present study findings 
Self-reported physical activity \& heat dissipation

indicate that aerobic fitness (as indexed by $\dot{\mathrm{V}} \mathrm{O}_{2 \text { peak }}$ alone) may be a better determinant of thermoregulatory function during exercise in the heat than PA level alone.

The observed similarities in WB-THL between the high and low PA groups may be attributed to 1) the workloads employed were of insufficient intensity and/or duration to reveal differences in heat loss; 2) the intensity of habitual PA performed by the high PA group was insufficient to induce a level of heat adaptation that would elicit differences in the body's physiological capacity to dissipate heat and/or 3) the two questionnaires used in the present study may not have offered the sensitivity required to detect potential differences in thermal adjustments associated with regular endurance activity in the current sample, despite being previously validated and commonly employed (Kohl et al. 1988; Baecke et al. 1982). Indeed, it is possible that the questionnaires employed may better reflect training-induced adjustments in

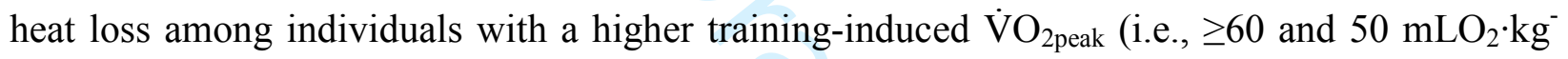
${ }^{1} \cdot \mathrm{min}^{-1}$ for young men and women, respectively). Thus, in context of our findings, we show that the use of PA questionnaires alone would be inadequate to assess an individual's capacity (or ability) to perform exercise in the heat (even among individuals that self-report a high level of PA). Future research using more objective measurements of background PA are therefore recommended to obtain a better understanding of how training-induced heat adaptation influences heat loss independently of $\dot{\mathrm{VO}}_{2 \text { peak }}$.

\section{CONCLUSION}

We show that self-reported PA level does not modulate the body's physiological capacity to dissipate heat independently from $\dot{\mathrm{VO}}_{2 \text { peak }}$ in young adults when exercising in a hot, dry environment. However, further research, involving individuals possessing high and low PA 
Self-reported physical activity \& heat dissipation

levels with more homogeneous secondary characteristics (e.g., body morphology, $\mathrm{V}_{2 \text { peak }}$ ) within each group, which may incorporate wearable technology for the monitoring of frequency and intensity of background PA and more robust measures of training-induced thermal adaptation, is required to better evaluate how habitual PA level may modulate heat loss.

\section{ACKNOWLEDGEMENTS}

This research was supported by the Ontario Ministry of Labour (views expressed in the publication are those of the authors and do not necessarily reflect those of the Ministry) and the Natural Sciences and Engineering Research Council of Canada (RGPIN-06313-2014, RGPAS462252-2014).

\section{AUTHOR CONTRIBUTIONS}

D.T.L. performed experiments and drafted the manuscript; all authors contributed to the concept and design of research, interpreted results of experiments as well as edit, revise, and approved the final version of the manuscript. No conflict of interest, financial or otherwise, are declared by the author(s). 
Self-reported physical activity \& heat dissipation

\section{REFERENCES}

Amano, T., Koga, S., Inoue, Y., Nishiyasu, T., and Kondo, N. 2013. Characteristics of sweating responses and peripheral sweat gland function during passive heating in sprinters. Eur. J. Appl. Physiol. 113: 2067-2075. Doi: 10.1007/s00421-013-2651-8. PMID: 23579362.

Avellini, B.A., Shapiro, Y., Fortney, S.M., Wenger, C.B., and Pandolf, K.B. 1982. Effects on heat tolerance of physical-training in water and on land. J. Appl. Physiol. 53: 1291-1298. doi:10.1152/jappl.1982.53.5.1291. PMID: 7174421.

Baecke, J.A., Burema, J., and Frijters, J.E. 1982. A short questionnaire for the measurement of habitual physical activity in epidemiological studies. Am. J. Clin. Nutr. 36: 936-942. doi: 10.1093/ajcn/36.5.936. PMID: 7137077.

Bouchard, C., An, P., Rice, T., Skinner, J.S., Wilmore, J.H., Gagnon, J., et al. 1999. Familial aggregation of $\operatorname{VO}(2 \max )$ response to exercise training: results from the HERITAGE Family Study. J. Appl. Physiol. 87: 1003-1008. doi: 10.1152/jappl.1999.87.3.1003. PMID: 10484570.

Henane, R., Flandrois, R., and Charbonnier, J.P. 1977. Increase in sweating sensitivity by endurance conditioning in man. J. Appl. Physiol. 43: 822-828. doi: 10.1152/jappl.1977.43.5.822. PMID: 591475.

Kohl, H.W., Blair, S.N., Paffenbarger, R.S., Macera, C.A., and Kronenfeld, J.J. 1988. A mail survey of physical activity habits as related to measured physical fitness. Am. J. Epidemiol. 127: 1228-1239. PMID: 3369421.

Lamarche, D.T., Notley, S.R., Louie, J.C., Poirier, M.P., and Kenny, G.P. 2017a. Fitness-related differences in the rate of whole-body evaporative heat loss in exercising men are heat-load dependent. Ex. Physiol. 103: 101-110. doi: 10.1113/EP086637. PMID: 29052285 
Self-reported physical activity \& heat dissipation

Lamarche, D.T., Notley, S.R., Poirier, M.P., and Kenny, G.P. 2017b. Fitness-related differences in the rate of whole-body total heat loss in exercising young healthy women are heat-load dependent. Ex. Physiol. 103: 312-317. doi: 10.1113/EP086752. PMID: 29250845.

Périard, J.D., Racinais, S., and Sawka, M.N. 2015. Adaptations and mechanisms of human heat acclimation: applications for competitive athletes and sports. Scand. J. Med. Sci. Sports. 25: 2038. doi: 10.1111/sms.12408. PMID: 25943654.

Saltin, B. and Hermansen, L. 1966. Esophageal, rectal, and muscle temperature during exercise.

J. Appl. Physiol. 21: 1757-1762. doi: 10.1152/jappl.1966.21.6.1757. PMID: 5929300. 
Self-reported physical activity \& heat dissipation

\section{TABLES}

Table I. Physical characteristics.

\begin{tabular}{cccccccc}
\hline Group & $\begin{array}{c}\text { Age } \\
(\mathrm{yr})\end{array}$ & $\begin{array}{c}\mathrm{Height} \\
(\mathrm{cm})\end{array}$ & $\begin{array}{c}\text { Body } \\
\text { mass } \\
(\mathrm{kg})\end{array}$ & $\begin{array}{c}\text { Body } \\
\text { surface area } \\
\left(\mathrm{m}^{2}\right)\end{array}$ & $\begin{array}{c}\mathrm{VO}_{2 \text { peak }} \\
\left(\mathrm{mLO}_{2} \cdot \mathrm{kg}^{-}\right. \\
\left.\mathrm{min}^{-1}\right)\end{array}$ & $\begin{array}{c}\text { Body fat } \\
(\%)\end{array}$ & $\begin{array}{c}\text { Level of physical activity, } \\
(\text { Intensity, minutes per } \\
\text { week })\end{array}$ \\
\hline High-PA $(n=10)$ & $23 \pm 4$ & $169 \pm 8$ & $68 \pm 9$ & $1.77 \pm 0.14$ & $38.5 \pm 2.7$ & $21 \pm 5$ & high-medium, $369 \pm 303$ \\
$\quad$ range (max-min) & $(30-19)$ & $(181-156)$ & $(82-50)$ & $(2.00-1.55)$ & $(42.8-34.3)$ & $(30-15)$ & $(840-180)$ \\
Low-PA $(n=10)$ & $21 \pm 2$ & $171 \pm 8$ & $67 \pm 9$ & $1.78 \pm 0.15$ & $35.9 \pm 5.3$ & $21 \pm 5$ & medium-low, $88 \pm 52 \dagger$ \\
range (max-min) & $(25-19)$ & $(183-160)$ & $(81-52)$ & $(2.01-1.54)$ & $(44.0-26.5)$ & $(27-15)$ & $(180-0)$ \\
\hline
\end{tabular}

Note: Values are means $\pm \mathrm{SD}$. $\dot{\mathrm{VO}}_{2 \text { peak }}$, rate of peak oxygen consumption. $\dagger$; Significant difference from High-PA. 
Self-reported physical activity \& heat dissipation

Table II. Thermal and physiological variables.

\begin{tabular}{|c|c|c|c|c|}
\hline & Baseline & Ex1 & Ex2 & Ex3 \\
\hline \multicolumn{5}{|l|}{$\mathrm{M}-\mathrm{W}, \mathrm{W} \cdot \mathrm{m}^{-2}$} \\
\hline High-PA & $55 \pm 4$ & $146 \pm 8$ & $194 \pm 9$ & $250 \pm 12$ \\
\hline Low-PA & $51 \pm 5$ & $150 \pm 6$ & $196 \pm 8$ & $246 \pm 11$ \\
\hline \multicolumn{5}{|l|}{ WB-EHL, $\mathrm{W} \cdot \mathrm{m}^{-2}$} \\
\hline High-PA & $69 \pm 10$ & $174 \pm 9$ & $213 \pm 12$ & $246 \pm 13$ \\
\hline Low-PA & $64 \pm 15$ & $171 \pm 11$ & $211 \pm 13$ & $238 \pm 20$ \\
\hline \multicolumn{5}{|l|}{ WB-DHE, $\mathrm{W} \cdot \mathrm{m}^{-2}$} \\
\hline High-PA & $-31 \pm 4$ & $-37 \pm 4$ & $-38 \pm 6$ & $-39 \pm 7$ \\
\hline Low-PA & $-36 \pm 6$ & $-41 \pm 6$ & $-40 \pm 6$ & $-41 \pm 7$ \\
\hline \multicolumn{5}{|l|}{$\mathrm{T}_{\text {eso }},{ }^{\circ} \mathrm{C}$} \\
\hline High-PA & $37.06 \pm 0.18$ & $37.37 \pm 0.21$ & $37.65 \pm 0.22$ & $38.09 \pm 0.24$ \\
\hline Low-PA & $37.13 \pm 0.21$ & $37.47 \pm 0.20$ & $37.80 \pm 0.29$ & $38.23 \pm 0.32$ \\
\hline \multicolumn{5}{|l|}{$\mathrm{T}_{\mathrm{sk}},{ }^{\circ} \mathrm{C}$} \\
\hline High-PA & $35.73 \pm 0.25$ & $35.87 \pm 0.26$ & $36.01 \pm 0.27$ & $36.31 \pm 0.30$ \\
\hline Low-PA & $35.54 \pm 0.34$ & $35.95 \pm 0.23$ & $36.18 \pm 0.26$ & $36.47 \pm 0.28$ \\
\hline \multicolumn{5}{|l|}{ Heart rate, bpm } \\
\hline High-PA & $89 \pm 7$ & $115 \pm 10$ & $136 \pm 11$ & $158 \pm 11$ \\
\hline Low-PA & $90 \pm 11$ & $124 \pm 14$ & $147 \pm 14$ & $167 \pm 12$ \\
\hline
\end{tabular}

Note: Values are means $\pm 95 \%$ confidence interval. $\mathrm{M}-\mathrm{W}$, metabolic heat production; WB-EHL, wholebody evaporative heat loss; WB-DHE; whole-body dry heat exchange (where negative values indicate dry heat gain in the conditions tested); $\mathrm{T}_{\text {eso }}$; esophageal temperature; $\mathrm{T}_{\mathrm{sk}}$, mean skin temperature. No betweengroup differences were realized $(P>0.05)$. 
Self-reported physical activity \& heat dissipation

\section{FIGURE CAPTION}

Figure I. Metabolic heat production (horizontal dashed black line) and whole-body total heat loss (circles) normalized for body surface area as well as the cumulative (total) change in body heat storage (excluding recovery) (Insert) in young adults with high and low levels of selfreported physical activity at baseline and during three 30-min exercise bouts (Ex1, Ex2, Ex3) (vertical dashed black lines) and three 15-min recovery bouts (R1, R2, R3) in a hot, dry environment $\left(40^{\circ} \mathrm{C}, 10 \%\right.$ relative humidity). Metabolic heat production is presented as the mean across groups, while heat loss and heat storage are expressed as the mean $( \pm 95 \%$ confidence interval) within each group. No between-group differences were realized $(P>0.05)$. 


\section{Figure I}

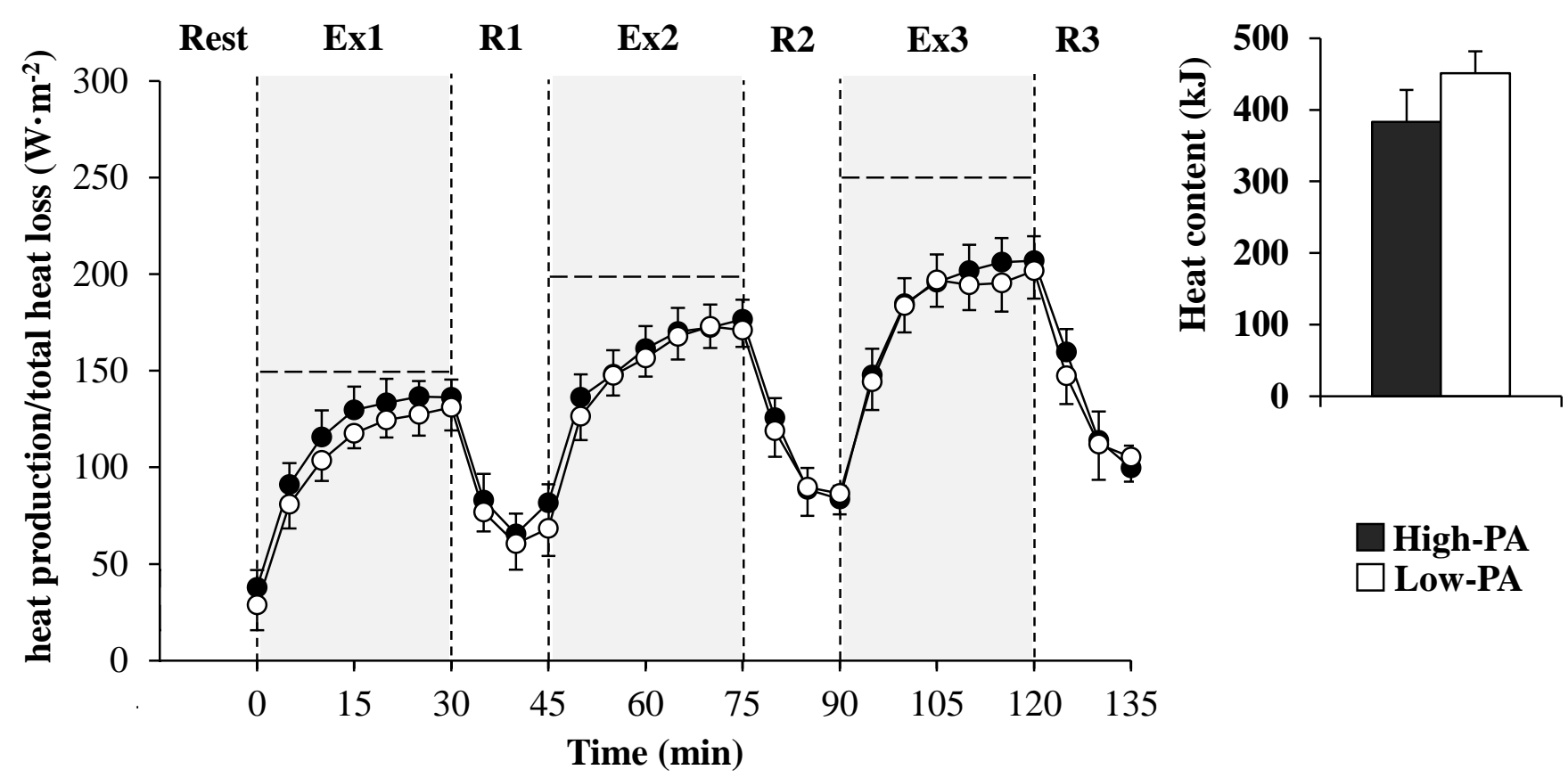

